RUNNING HEAD: Temporal Dynamics of Attentional Bias

\title{
Temporal Dynamics of Attentional Bias
}

\author{
Zvielli, Ariel, M.A. \\ Bernstein, Amit, Ph.D. \\ University of Haifa, Israel \\ Koster, Ernst H.W., Ph.D. \\ University of Ghent, Belgium
}

Corresponding Author: Amit Bernstein, PhD; Director, The International Research Collaborative on Anxiety. Dr. Bernstein can be contacted at the University of Haifa, Department of Psychology, Mount Carmel, Haifa, 31905, Israel, 972-4-828-8863 (phone), 972-4-824-0966 (facsimile). Electronic mail: abernstein@psy.haifa.ac.il

Author Note: Conceptualization of the reported Trial Level Bias Score (TL-BS) approach, computational methodology, programming, and data analysis were conducted by Dr. Amit Bernstein and doctoral fellow Ariel Zvielli in the International Research Collaborative laboratory at the University of Haifa, Israel. All authors wrote the paper. Study 1 data were collected by An Raes at Ghent University, Belgium. Study 2 data were collected in Dr. Bernstein's laboratory at the University of Haifa. Researchers interested in the application of the proposed Trial Level Bias Score (TL-BS) analysis methodology in their research are welcome to contact the corresponding author (A. Bernstein) for assistance. 


\begin{abstract}
Biases of emotional attention $(\mathrm{AB})$ are believed to be central to human (mal)adaptation and multiple forms of psychopathology. Yet, fundamental questions remain regarding the nature and empirical study of $\mathrm{AB}$. We thus aimed to: (1) test a novel conceptualization and related operationalization of $\mathrm{AB}$ expression in time; and (2) illuminate the nature of $\mathrm{AB}$ and specifically its temporal expression. We examined $\mathrm{AB}$ expression in time by means of a novel Trial Level Bias Score (TL-BS) analysis of dot probe task data in two experiments - among spider phobics and healthy controls, and among smokingdeprived daily smokers. Findings revealed evidence of the dynamic expression of $A B$ in time; furthermore, TL-BS parameters demonstrated unique associations with psychopathology and addiction beyond traditional bias score. The present research may help to bring the conceptualization and quantification of $\mathrm{AB}$ closer to the nature of the phenomenon and thereby advance basic and clinical knowledge.
\end{abstract}

Key Words: Addiction; Anxiety; Attentional Bias; Attention Paradigms; Dot Probe; Emotional Attention; Information Processing; Motivation; Phobia; Reward; Smoking; Substance Use; Threat 


\section{Temporal Dynamics of Attentional Bias}

\section{General Introduction}

Selective attention to appetitive and aversive stimuli is important to human adaptation (Bar-Haim et al., 2010; Cisler \& Koster, 2010; Robinson, Charney, Overstreet, Vytal, \& Grillon, 2012). Due to the functional importance of early and fast processing of motivationally-relevant information, theories have argued that selective attention will be allocated to emotionally-evocative information (Lang, Bradley, \& Cuthbert, 1997; Öhman, Flykt, \& Lundqvist, 2000). Dysregulation in this “emotional attention” process - termed Attentional Bias (AB) - has been linked to a cascade of information processing biases and behavior underlying the development and maintenance of multiple forms of psychopathology and addiction (Bar-Haim, Lamy, Pergamin, Bakermans-Kranenburg, \& van IJzendoorn, 2007; Cisler \& Koster, 2010; Luijten et al., 2012; Mathews \& MacLeod, 2005; Osinsky, Lösch, Hennig, Alexander, \& MacLeod, 2012; Shechner et al., 2012). For example, threat-related AB has been linked to anxiety disorders (Bar-Haim et al., 2007; Van Bockstaele et al., 2014), AB for self-related information has been linked to depression (De Raedt \& Koster, 2010), and AB for drug cues has been linked to addiction and substance abuse (Field, Munafò, \& Franken, 2009). Accordingly, information processing biases and

attentional biases specifically represent key etiological and maintenance factors in central theories of prevalent mental disorders (e.g., social anxiety: Clark \& Wells, 1995; depression: De Raedt \& Koster, 2010).

In recent years, emotional attention and $\mathrm{AB}$ has emerged as an important research area of cognitive-affective science and basic and clinical psychopathology research - with hundreds of studies published annually. Most of these studies rely on modifications of well-investigated cognitiveexperimental tasks such as probe detection, spatial cueing, and visual search where behavioral response 
latencies (i.e., reaction time, RT) permit inferences regarding allocation of covert attention to "emotional" or motivationally-relevant information (Pashler, 1998; Weierich \& Barrett, 2010). Similarly, other recent work has also evaluated overt attention (i.e., eye movement data; Armstrong \& Olatunji, 2012; Bradley, Mogg, \& Millar, 2000). Furthermore, scholars have increasingly attempted to understand the genetic and learning bases of ABs (Beevers, Wells, Ellis, \& McGeary, 2009; Fox, Zougkou, Ridgewell, \& Garner, 2011; Perez-Edgar et al., 2010) as well as their neural substrate (Browning, Holmes, Murphy, Goodwin, \& Harmer, 2010; Eldar, Yankelevitch, Lamy, \& Bar-Haim, 2010; Monk et al., 2006). This domain of research reflects the ongoing field-wide search for malleable etiological and maintaining bio-psycho-behavioral processes that cut across psychiatric disorders and thus may be candidate targets for novel intervention approaches (Insel et al., 2010).

Despite this body of work, theory, and clinical intervention development, the current $\mathrm{AB}$ literature is - on a number of fronts - grappling with the nature of this process and its operationalization. First, the measurement of $\mathrm{AB}$ using RT measures such as the dot probe task (MacLeod, Mathews, \& Tata, 1986) has shown poor split-half and test-retest reliability (Schmukle, 2005); and questions regarding the psychometrics of these tasks are emerging as a new frontier for this area of research more broadly (e.g., Cisler, Bacon, \& Williams, 2009; Dear, Sharpe, Nicholas, \& Refshauge, 2011). Moreover, correlations between RT measures of $\mathrm{AB}$ are generally very low (e.g., $\mathrm{r} \sim .00$ - .20; Brosschot, de Ruiter, \& Kindt, 1999; Dalgleish et al., 2003; Mogg et al., 2000). Similarly, there is substantial heterogeneity and mixed findings reported on $\mathrm{AB}$ and its correlates and outcomes (for a recent review, Van Bockstaele et al., 2014). Indeed, despite the central role of informational processing biases, and attentional biases specifically, in prominent theories of psychopathology, empirical data as to the role of attentional biases in these disorders are mixed, and at best demonstrate small to moderate effects (Bar-Haim et al., 2007; Van Bockstaele et al., 2014). Furthermore, efforts to therapeutically target attentional biases - such as 
Attentional Bias Modification (ABM) have similarly demonstrated mixed findings, and when effective, small effects (e.g., Beard, Sawyer, \& Hofmann, 2012; Hallion \& Ruscio, 2011; Van Bockstaele, Koster, Verschuere, Crombez, \& De Houwer, 2012). Moreover, in anxiety disorders there is extensive debate whether $\mathrm{AB}$ is related to vigilance and/or difficulties to disengage attention from threat (Bar-Haim et al., 2007; Cisler \& Koster, 2010; Clarke, Chen, \& Guastella, 2012; Clarke, MacLeod, \& Guastella, 2013; Eysenck, Derakshan, Santos, \& Calvo, 2007; Fox, Russo, Bowles, \& Dutton, 2001; Sheppes, Luria, Fukuda, \& Gross, 2013; Yiend \& Mathews, 2001). Additionally, in some instances - when threat is presented longer or actual threat is present - attentional avoidance from threat is observed (Koster, Crombez, Verschuere, Van Damme, \& Wiersema, 2006; Mogg, Bradley, Miles, \& Dixon, 2004; Pflugshaupt et al., 2005; Wilson \& MacLeod, 2003). Thus, the time-course of the expression of emotional attention is a growing focus of research (e.g., Cooper \& Langton, 2006; Mogg \& Bradley, 2006).

Recently, Zvielli, Bernstein and Koster (2014) found that when examining covert AB for different categories of threatening information (e.g., threatening -animals, -faces, violence), anxious adults demonstrated multiple patterns of $\mathrm{AB}$ for threatening stimuli. $34 \%$ of participants expressed $\mathrm{AB}$ towards threat stimuli, 20.8\% AB away from threat stimuli, and 34\% AB towards some categories of threat stimuli and away from others, and the remaining no $\mathrm{AB}$. In contrast to views wherein $\mathrm{AB}$ is conceptualized as a stable individual difference variable (e.g., Mogg \& Bradley, 1998; Williams, Watts, MacLeod, \& Mathews, 1988), these latter findings may point to variability across and within participants in the expression of $\mathrm{AB}$ - or a more dynamic perspective regarding the expression of $\mathrm{AB}$. One could argue that the varied findings and measurement problems indicate that these paradigms and/or behavioral RT data are simply unreliable - that the problem is the paradigm itself. We argue that neither the tasks nor behavioral RT data are to necessarily blame. Rather, work to-date has relied on 
what may be a suboptimal way to map and analyze the possible dynamic nature of emotional attention because of our conceptualization of the phenomenon.

We theorize that these questions and mixed findings may be, in part, accounted for by an important limitation in extant theory and empirical study of the nature of the expression of $A B$. Indeed, normative and dysregulated selective attention is a psychological process expressed repeatedly and continuously over time. Accordingly, in various methodological paradigms [e.g., the dot probe (MacLeod et al., 1986), spatial cueing (Fox et al., 2001), emotional stroop (for a review, see Williams, Mathews, \& MacLeod, 1996), visual search (Wolfe, 1994), anti-saccade (Hallett, 1978)] AB is sampled repeatedly over time, in temporally contiguous trials. In work to-date, quantification of $\mathrm{AB}$ may not optimally reflect the time-series expression and measurement of AB. To illustrate this idea, we focus on the most commonly used paradigm to study selective spatial emotional attention and $\mathrm{AB}$, the dot probe task (MacLeod et al., 1986).

In the dot probe paradigm participants are instructed to focus on a centrally presented fixation cross, then stimuli (images, words) are briefly (typically 250-750ms) presented at the top and bottom (alternatively, right and left) of a monitor. After stimuli offset, a probe appears in location of one of the two stimuli. Participants are asked to indicate the location/features of the probe by pressing one of two buttons (top vs bottom/left vs right, or alternatively are asked to discriminate between different probes (e.g., "e" of "f"). The core idea is that selective attention towards a motivationally relevant or emotional stimulus will speed reaction time when a probe appears in the spatial location of the target stimulus and slow RT when a probe appears in the spatial location opposite of the target stimulus. Bias scores (BS) are computed by subtracting Mean RT of Congruent Trials (CT; i.e., trials in which the probe replaces a target cue) from Mean RT of Incongruent Trials (IT; i.e., in which the probe replaces the neutral (not target) cue (NTs); (Bar-Haim et al., 2007). Thus, AB is inferred from differences in RTs in congruent 
trials (CTs) (e.g., aversive, appetitive), relative to incongruent trials (ITs): AB towards target stimuli is inferred when IT > CT and $\mathrm{AB}$ away from target stimuli is inferred when $\mathrm{CT}>\mathrm{IT}$. One empirical corollary justifying this quantification of $A B$ is that the temporal order of CTs and ITs is not relevant; the theoretical assumption grounding this empirical corollary is that $\mathrm{AB}$ is expressed in a stable or static manner on all emotional-neutral trial pairs. Put differently, it is thought that each trial reflects an equivalent sample from the same underlying stable trait-like signal of $\mathrm{AB}$. A second related corollary justifying extant quantification of $A B$ is that each person may either express $A B$ towards or away from motivationally-relevant stimuli. Yet, research to-date has not directly tested these assumptions nor attempted to evaluate the expression of $\mathrm{AB}$ concurrent with its expression in time. Moreover, seminal studies in the context of emotion processing and smoking already pointed to the possibility of carry-over effects in attentional bias measures (emotional stroop) and thus argued against the use of simple mean AB scores (McKenna \& Sharma, 2004; Waters, Sayette, \& Wertz, 2003).

We thus propose that the current conceptualization and related quantification of AB may not optimally reflect the time-series expression and measurement of $\mathbf{A B}$. We theorize that this mismatch between (a) the expression and measurement of $\mathrm{AB}$ in time, on the one hand, and (b) its conceptualization and related quantification independent of time, on the other hand, has hindered the field's study and understanding of AB. Accordingly, we propose that potentially important features of $\mathrm{AB}$ expression have failed to receive theoretical or empirical attention due to our failure to conceptualize and quantify $\mathrm{AB}$ concurrent with its real-time expression. Specifically, we theorize that emotional attention or AB may not be expressed as a stable or static "signal". Rather, we theorize that AB may be expressed in fluctuating, phasic bursts, towards and/or away from target stimuli over time. Basic neuroscience of attention, vision and eye movement data may be interpreted to support the hypothesis that $\mathrm{AB}$ is a dynamic and fluctuating process (e.g., Ardid, Wang, Gomez-Cabrero, \& 
Compte, 2010; Harris \& Pashler, 2004; Herrmann \& Knight, 2001). Furthermore, the proposed dynamic expression of emotional attention may be expected due to the possible role of various variables that may act upon attention allocation and control (Desimone \& Duncan, 1995; Eysenck et al., 2007).

Proposed Trial-Level Computation of Attentional Bias. Using extant methodological paradigms (i.e., dot probe), we propose a novel means to analyze and study $\mathrm{AB}$ concurrent with its repeated, realtime expression. First, we propose a novel Trial Level Bias Score (TL-BS) computational methodology. TL-BS is computed by subtracting temporally contiguous pairs of CT and IT response times (RTs). Specifically, each IT is matched, with a CT that is temporally as close as possible and no further than 5 trials ${ }^{\mathrm{i}}$ away (before or after) from the IT. The same method is applied to match each CT to its temporally contiguous IT. This approach produces a time-series of TL-BSs per participant. ${ }^{\mathrm{ii}}$ TL-BS is thus a sequential, signal-like series of IT-CT difference scores that reflect AB estimations in time concurrent with the expressed repeated allocation of attention. Importantly, the computation of TL-BS requires no change in the standard use of the Dot-Probe task, as the input for the calculation is simply RTs and trial type (CT/IT) as in the traditional mean BS estimation.

Figure 1 shows an example of TL-BSs for two participants - a spider phobic participant and healthy control participant (illustration from Experiment 1 data). TL-BS may be further quantified to parameters that may capture novel dynamic features of $\mathrm{AB}$ expression, and thus allow empirical tests of TL-BS significance and importance. These types of parameters of TL-BS may include: individual differences in phasic bursts or "peaks" of AB expressions; mean levels of TL-BS towards and away from target stimuli; degree of TL-BS variability in time, across the spectrum of AB (towards, away or both).

Studies Overview \& Aims 
First, we examined whether AB could be meaningfully operationalized using TL-BS to explore the expression of $\mathrm{AB}$ to aversive and appetitive stimuli. In doing so we furthermore aimed to examine whether the traditional mean $\mathrm{BS}$ computation of $\mathrm{AB}$ is a valid reflection of a stable or static signal of AB either towards or away from motivationally-relevant stimuli. Second, we aimed to examine whether novel TL-BS parameters of AB predict key features of psychopathology and addiction consistent with theory. Specifically, we tested whether TL-BS parameters of AB, relative to the traditional computation of $\mathrm{AB}$, predict diagnostic status (Experiment 1) and substance use rates (Experiment 2). These aims were addressed in two empirical studies. First, in Experiment 1, we evaluated $\mathrm{AB}$ to spider stimuli among spider phobics and healthy controls. Second, in Experiment 2, we manipulated the appetitive motivational relevance of smoking by depriving regular smokers of smoking for 18-hours (biochemically verified abstinence) and measured $A B$ to smoking stimuli.

\section{EXPERIMENT 1}

\section{Introduction}

$\mathrm{AB}$ has been studied and demonstrated most extensively in the anxiety disorders (Bar-Haim et al., 2007; Van Bockstaele et al., 2014). Thus, in Experiment 1, we tested TL-BS with respect to spider stimuli among spider phobics and healthy controls. The anxiogenic stimuli relevant to the specific clinical population provide a strong experimental context to rigorously test the novel hypothesis about $\mathrm{AB}$ temporal dynamics. Indeed, in contrast to more heterogeneous clinical populations (e.g., Generalized Anxiety Disorder) or threat-related stimuli, extant theory would predict a stable or static signal of $\mathrm{AB}$ in this sample.

Hypotheses. First, we hypothesized that TL-BS will not be consistent with a static trait-like signal over time. We expected that TL-BS will show phasic bursts of differential attentional allocation over time; and fluctuating temporal variability, ranging from towards and/or away from spider images 
over time. Second, we hypothesized greater levels of TL-BS towards spider stimuli will discriminate between spider phobics and healthy controls (Lavy, Van den Hout, \& Arntz, 1993; Öhman \& Mineka, 2001; Öhman, Flykt, \& Esteves, 2001). Theoretically, participants phobically fearful of spiders will exhibit hypervigilance to spider cues and may have difficulty disengaging attention from those cues. In contrast, healthy controls may also look towards spider images relative to neutral stimuli, but may be less hypervigilant and/or more able to disengage their attention from those stimuli in order to identify probe location. Third, we expected that elevated dysregulation in $\mathrm{AB}$ expression - characterized by temporal variability in levels of TL-BS - will discriminate between phobic participants and healthy controls. Theoretically, attentional dyscontrol underlying $\mathrm{AB}$ to phobic stimuli may lead to occasional bursts of TL-BS towards spiders followed by inhibitory or down-regulatory efforts to avoid extended exposure to phobic stimuli, followed again by a phasic burst of TL-BS towards spiders, etc. Such temporal dynamics may reflect attentional dyscontrol (S. J. Bishop, 2008; S. Bishop, Duncan, Brett, \& Lawrence, 2004; Derryberry \& Reed, 2002; Eysenck et al., 2007) or controlled efforts to regulate emotional responding (i.e., re-adjust attentional allocation; Heeren, De Raedt, Koster, \& Philippot, 2013; Siegle, Ghinassi, \& Thase, 2007).

\section{Method}

\section{Participants}

Sixty-six participants $\left(M(S D)_{a g e}=21.9(5.7) ; 87.5 \%\right.$ female $)$ were recruited via advertisements online at the website of Ghent University, via posters in the community, and via acquaintances. Spider fearful individuals were specifically invited to participate and only participants fulfilling the criteria for spider phobia were included $(\mathrm{N}=41)$. A healthy control group was similarly recruited $(\mathrm{N}=25)$. At the beginning of the baseline session, all participants were screened for spider phobia with the Dutch version of the Anxiety Disorders Interview Schedule for DSM-IV (ADIS-IV; Brown, DiNardo, \& Barlow, 
1994; Dutch translation by Bouman, de Ruiter, \& Hoogduin, 1995). Based on this interview, one participant did not meet the criteria for spider phobia and was excluded. All participants were at least 18 years of age and had not received previous treatment for spider phobia.

\section{Procedure}

All participants completed written informed consent. Participants who self-identified as spider fearful were fully assessed (i.e., those that do not endorse fear of spiders cannot qualify for spider phobia diagnostic status; American Psychiatric Association, 2013). At first, they were presented with the FSQ. Following that, spider phobia was assessed using the animal phobia section of the ADIS-IV-NL. The control group also filled out the FSQ. After the assessment phase all participants completed the dot probe task. Additional measures were collected but not reported, as these yet to be reported dot probe data were collected as part of a larger treatment study (Raes, Koster, Loeys, \& De Raedt, 2011).

Attentional Bias Measurement. The visual emotional dot probe task (MacLeod et al., 1986; Mogg et al., 2000) was used to measure AB. Participants were presented with a fixation cross (500ms), followed by $250 \mathrm{~ms}$ blank screen, followed by two stimuli presented simultaneously for a duration of either 200 or $500 \mathrm{~ms}$. One stimulus was presented to the left of the fixation cross and the other to the right, one of which was immediately replaced by a small black probe that was randomly presented to the right or left. Participants were instructed to first focus their gaze on the fixation cross and then, as quickly and accurately as possible, press one of two (left or right) response box buttons using their two index fingers corresponding to the location of the probe. A random interval of 750 , 1000, or $1250 \mathrm{~ms}$ preceded the next trial. On incongruent trials (IT), the probe appeared in the location of the neutral (butterfly) stimulus, whereas on congruent trials (CT) the probe appeared in the location of a spider stimulus. Additional trials include neutral stimuli presentation (i.e., trial with one dog and one rabbit; NT). Two blocks of trials $(200 \mathrm{~ms}, 500 \mathrm{~ms})$ were counter-balanced. Each block started with a single 
practice trial, followed by 60 experimental trials, of which 20 were NTs, 20 CTs and 20 ITs, randomly distributed across the block. Moreover, to increase semantic processing of the pictures, participants were instructed to press the space bar when presented with a picture of a fish. Each block contained 10 of these surprise trials. Target stimuli were 20 color photographs of spiders. Each picture was paired with a photograph of a butterfly matched as closely as possible for complexity, color, and luminescence. An additional set of 10 picture pairs of rabbits and dogs, were used as NTs.

\section{Materials \& Apparatus}

Questionnaires. The Fear of Spiders Questionnaire (FSQ; Szymanski \& O'Donohue, 1995) entails 18-items each scored on a 1-7 Likert-type scale $(1=$ does not apply to me, $7=$ applies very much to me). The FSQ has high internal consistency and good test-retest stability, and adequately discriminate phobic from non-phobic populations (Muris \& Merckelbach, 1996).

Stimuli. Pictures were digitized and adjusted in size so that each was 60 (height) x 80 (width) mm when displayed on the screen. When presented side by side on the screen, the distance between the inner edges of the pictures in each pair was $9 \mathrm{~cm}$.

Experimental set-up. The experiment was run via Inquisit presentation software (Inquisit 2.0). The experimental session was conducted in an acoustically-insulated room, with a one-way observation window. Participants were seated with their eyes $\sim 60 \mathrm{~cm}$ from the screen. Participants' responses were measured via standard keyboard.

\section{Results}

Spider Phobia. FSQ scores among spider phobic participants $(M(S D)=91.39(20.62))$ were significantly elevated relative to the control participants $\left(M(S D)=21.87(22.21) ; t_{(62)}=12.59, p<.001, d\right.$ $=3.2)$. 
Data preparation. RT of outlier trials (trial RT $<200$ or $>1500 \mathrm{~ms}$, trial RT $>$ or $<3$ SDs of participant's mean RT) or errors (i.e., "left" response when probe appeared on the right) were discarded. In both $500 \mathrm{~ms}$ and $200 \mathrm{~ms}$ blocks, $M(S D) \%$ of outlier trials $=0.10(0.34) \%$ and $M(S D) \%$ error trials $=2.23(3.17) \%$.

Traditional Computation of Attentional Bias. Traditional Bias Score (BS) was computed by subtracting mean CT from mean IT RTs (MacLeod \& Mathews, 1988). Descriptive statistics for Traditional BS by group and stimuli exposure duration are presented in Table 1.

Trial-Level Bias Scores: Computation. We computed TL-BS by first matching and then subtracting temporally contiguous pairs of CT and IT response times by subject - see detailed description in Introduction and Figure 1. Out of 20 ITs and 20 CTs, the number of computed TL-BSs per participant in $500 \mathrm{~ms}$ and $200 \mathrm{~ms}$ blocks was $M(S D)=36.7(2.3)$, Range $=24$ to 40 . The number of sequential trials with no calculation of TL-BS: $M(S D)=1.8(.13)$, Range $=1-17$. The number of trials between paired IT-CT trials was $M(S D)=1.78(1.01)$.

We visually inspected TL-BS within and across participants (see Figure 2). As predicted, AB appears to be expressed in phasic bursts or peaks over time. Furthermore, the magnitude and direction of peaks vary within- and between-participants, as does the degree of temporal variability.

Trial-Level Attentional Bias: Quantitative Parameters. As described in the Introduction, computation of TL-BS enables quantification of novel parameters of $\mathrm{AB}$ expression in time. Accordingly, based on our theorizing regarding the dynamics of $\mathrm{AB}$ expression, we identified 3 candidate parameters of trial-level $\mathrm{AB}$ that permit direct study of TL-BS or $\mathrm{AB}$ in time, comparison of TL-BS parameters between groups, relative to correlates, etc. These quantitative parameters were chosen as initial candidates reflecting theoretically-relevant individual differences in the expression of trial-level attentional bias, including: (1) Mean TL-BS. This parameter is bi-dimensional, and therefore calculated two-fold - (1a) Mean TL-BSPositive - Individual differences in degree to which mean TL- 
BS > 0ms or towards target stimuli; mean of TL-BSs > 0ms per participant; (1b) Mean TL-BS NEGATIVE $_{\text {. }}$

- Individual differences in degree to which TL-BS < 0ms or away from target stimuli; mean of TL-BSs $<0$ ms per participant. (2) Peak TL-BS. This parameter is also bi-dimensional, and therefore calculated two-fold - (2a) Peak TL-BS Positive - Individual differences in the maximum phasic expression of trial-

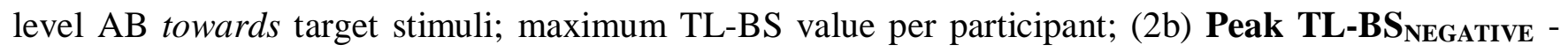
Individual differences in the maximum phasic expression of trial-level $\mathrm{AB}$ away from target stimuli; minimum TL-BS value per person. (3) Variability in TL-BS reflects the degree of stability or temporal variability in the expression of $\mathrm{AB}$ towards and/or away over time; sum of all distances between all sequential TL-BSs (i.e., length of TL-BS "line") divided by total number of TL-BSs. See Table 1 for descriptive data for traditional mean BS and TL-BS parameters.

Traditional and Trial-Level Attentional Bias Parameters and Phobia Diagnostic Status. To test hypotheses as to the capacity for TL-BS to discriminate between phobic and healthy control group status, we conducted 6 logistic regression analyses per stimulus presentation block (500ms/200ms). See Table 1 for detailed results of regression analyses. First, in the 500ms block, levels of traditional BS were marginally significantly related $(p=.07)$ to spider phobic group status. As predicted, higher levels

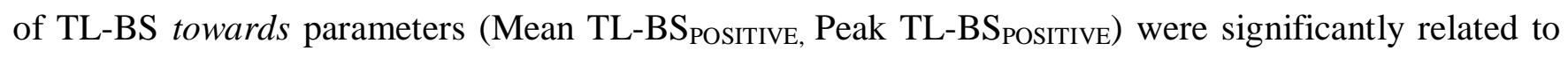
greater likelihood of spider phobic group status. In addition, as predicted, greater degree of Variability in TL-BS was significantly related to spider phobia diagnostic status. Furthermore, though not hypothesized, lower levels of mean TL-BS ${ }_{\text {NEGATIVE }}$ were also significantly related to greater likelihood of spider phobic group status. Second, for the $200 \mathrm{~ms}$ block, traditional BS was significantly related to spider phobic group status. With respect to TL-BS parameters, the same pattern of results observed for 500ms block were observed (Table 1). 
Furthermore, to determine whether each hypothesized TL-BS parameter (Mean TL-BS Positive, Peak TL-BS Positive, Variability in TL-BS) explained variance above and beyond traditional BS, we conducted 3 additional logistic regression analyses per stimulus duration block (500ms/200ms). Traditional BS was entered in step 1 and each of the TL-BS parameters was entered at step 2 of each regression equation. See Table1 for results of hierarchical logistic regression analyses. First, in the

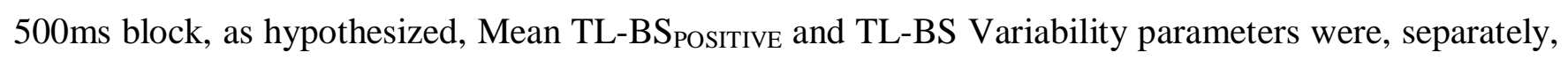
found to explain variance in spider phobic group status above and beyond the effect of traditional BS (Incremental Nagelkerke's $R^{2}=.04$ to .13); However, Peak TL-BS Positrive did not. Furthermore, traditional BS did not explain unique variance above and beyond the TL-BS parameters. Second, for the 200ms block, each of the TL-BS towards parameters explained variance above and beyond traditional BS (Incremental Nagelkerke's $r^{2}=.07$ to .13 ); and again, traditional BS did not explain unique variance above and beyond the TL-BS towards parameters. However, we found that both TL-BS Variability and traditional BS each explained unique incremental variance in spider phobic group status above and beyond the other (See Table 1).

Finally, to rule-out the possibility that the observed are a by-product of intra-individual differences and variability in RT - we ran three additional tests. Because TL-BS and traditional BS are computed by IT-CT differences, they are by definition related to IT RT and CT RT (i.e., due to multicollinearity). Thus we utilized RT on neutral trials as a marker of degree and variability of RT. First, as in past work in anxiety, we found that the mean RT on neutral trials distinguished between diagnostic groups - such that phobics were significantly slower than controls $\left(O R=1.11, C I_{95 \%}=1.03-1.20, p=\right.$ .01). Crucially, we found that TL-BS Variability explained unique variance above and beyond mean neutral RT in predicting group status $\left(O R=1.54, C I_{95 \%}=1.06-2.26, p=.01\right)$, and mean neutral RT did not explain significant variance above and beyond TL-BS Variability $\left(O R=1.04, C I_{95 \%}=0.94-1.15\right.$, 
n.s). However, the Positive TL-BS parameters (i.e., Peak, Mean) as well as traditional BS did not predict group status above and beyond neutral mean RT $(O R<1.1$, n.s). Finally, we wanted to add an additional, particularly rigorous test of the robustness of TL-BS and derived parameters. "Fake TL-BS" parameters were created using neutral trials' RTs. This was done by relating to neutral trials as if they were a random sequence of "CTs" or "ITs". We then used the same algorithm to compute "fake TL-BS" generating neutral trial pairs in a manner identical to our computation of TL-BS of real IT-CT pairs. As expected, the parameters derived from the "fake TL-BS" did not significantly predict diagnostic status rates $\left(O R<1.15, C I_{95 \%}=0.97-1.35\right.$, n.s $)$.

\section{Discussion Experiment 1}

We investigated the expression of attentional bias to motivationally-relevant spider stimuli in spider phobics and healthy controls in the modified dot probe task by means of the proposed TL-BS computational methodology. First, we found that AB may be operationalized by means of the TL-BS computational methodology in a manner that provides repeated $\mathrm{AB}$ estimates at the trial-level, temporally concurrent with the dynamic expressions of AB. Second, consistent with prediction, TL-BS data were not consistent with a static trait-like signal over time. TL-BS demonstrated phasic bursts of differential attentional allocation over time; as well as evidence of fluctuating temporal variability ranging from $\mathrm{AB}$ towards and/or away from spider images. Specifically, the amplitude and direction of peaks vary within- and between-participants, as does the degree of temporal variability. Third, we were able to derive initial candidate parameters reflecting the time-series trial-level $\mathrm{AB}$ expression over time including: degree of $\mathrm{AB}$ towards and/or away from target stimuli as well as degree of stability or temporal variability in the expression of $\mathrm{AB}$ towards and/or away over time.

The derived parameters permitted us to explore the criterion-related validity of the TL-BS operationalization of $\mathrm{AB}$ with respect to spider phobia diagnostic status. First, as hypothesized, levels of 
TL-BS POSITIVE parameters - reflecting AB towards spider stimuli - significantly discriminated between spider phobics and healthy controls. Second, as predicted, greater levels of temporal variability of AB discriminated between phobics and controls. Third, though not predicted, peak levels of TL-BS away from spider stimuli discriminated between diagnostic groups. The latter effect may be a by-product of the magnitude of the fluctuating temporal variability of $\mathrm{AB}$ expression (from towards to away from spider stimuli) - among phobics. This overall pattern of effects were observed both for the 500-ms and the 200-ms blocks. Importantly, these effects were observed above and beyond the effect of traditional mean BS. In contrast, traditional mean BS did not account for unique variance beyond most of the TLBS parameters. This pattern of TL-BS incremental effects was observed both in the 500ms and 200ms blocks. There were two exceptions to this pattern of TL-BS incremental effects. First, in the 500ms block, the Peak TL-BS POSITIVE did not account for unique variance beyond traditional mean BS; it was though incrementally significant in the 200ms block. Second, in the 200ms block (though not in the 500ms block) traditional mean BS also accounted for unique variance in diagnostic status beyond TLBS Variability.

Finally, though mean RT on neutral trials distinguished between groups (see also Mogg et al., 2008), the "fake TL-BS" parameters did not distinguish between groups. Thus, it is not likely that the TL-BS parameters may be accounted for by intra-individual variability in RT. The observed RT differences between phobics and non-phobics could have multiple bases. Mogg et al (2008) documented a slowdown in response time in the context of high anxiety. From this perspective, TL-BS parameters and traditional bias scores - and in fact any use of such paradigms relying on congruency effects - are similarly limited by the fact that phobic/anxious groups differ in overall RT even on neutral trials - as we observed. Furthermore, in the specific context of exposure to spider phobia studied here, anticipatory search on all trails including neutral trials (as they have the potential to be phobic stimulus trials) and 
carry-over effects between CT/IT trials to neutral trials are likely (see Waters et al., 2003). Phobic participants specifically are likely anticipating and searching for spiders more actively in visual space, slowing their overall RT to respond to probe location on all stimuli - including neutral stimuli. Overall, these data suggest that TL-BS estimates and parameters are not likely a by-product or proxy of general variability in RT. Note that we could not compute split-half reliability of the TL-BS parameters meaningfully due to the limited number of trials in this task. Specifically, task length (only 60 total trials of which 20 are neutral trials and thus not used in the TL-BS computations) was too short to permit meaningful computation of TL-BS parameters on half of the task trials (i.e., first set of $\sim 10$ CT-IT pairs derived from 20 trials and second set of $\sim 10$ CT-IT pairs derived from the other 20 trials) as is needed to compute split-half reliability. We thus examined reliability of the TL-BS parameters in Experiment 2.

\section{EXPERIMENT 2}

\section{Introduction}

In Experiment 2, we manipulated the appetitive motivational relevance of smoking stimuli by depriving regular smokers from smoking for 18-hours. This approach provides not only a test of TL-BS with respect to $\mathrm{AB}$ for appetitive cues, but does so in a controlled experimental context characterized by the acute motivational-relevance of the stimuli. As in Experiment 1, the controlled context-specific appetitive motivational-relevance of the stimuli provides a rigorous context to explore the stability of $\mathrm{AB}$ expression in time.

Hypotheses. First, we hypothesized that TL-BS among the deprived smokers will not be consistent with a static trait-like signal per traditional mean BS estimation; rather, we expected that TLBS will show the theorized phasic bursts of differential attentional allocation over time and fluctuating temporal variability. Second, we hypothesized that TL-BS towards-parameters would concurrently predict number of cigarettes smoked per day. This hypothesis is grounded in information processing 
theory of addiction, withdrawal, and cessation; wherein greater AB towards smoking cues is driven by and contributes to the motivation state potentiating smoking behavior and thus elevated rates of smoking over time (Baker, Piper, McCarthy, Majeskie, \& Fiore, 2004; Field et al., 2009; Waters et al., 2003). In contrast, smokers who demonstrate less $\mathrm{AB}$ towards smoking cues, even when deprived of smoking and thus experiencing withdrawal symptoms and related stress of deprivation, may less quickly or intensely express a motivational state to pursue use (i.e., to seek negative reinforcement opportunity). Accordingly, they are expected to smoke less frequently in their daily lives (Baker et al., 2004; Field et al., 2009).

\section{Method}

\section{Participants}

Forty-five $\left(M(S D)_{\mathrm{age}}=26.3(4.7) ; 42.2 \%\right.$ female $)$ adult daily smokers were recruited from the general community in the greater Haifa (Israel) metropolitan area to participate in a study on "emotions and smoking". Inclusion criteria were: a) 18 to 65 years of age; b) daily-smoker for at least the past year; c) currently (past-month) smoking at least 10 cigarettes per day; d) had not reduced the number of cigarettes by more than half in the past six-months. Exclusion criteria were: (a) possible pregnancy; (b) seizure disorder; (c) current or past cardiopulmonary illness (e.g., heart disease) or other major chronic illness (e.g., cancer); (d) current psychotic symptoms; (e) current nicotine replacement therapy or Zyban; (f) current use of other tobacco products more than once a week; (g) current homicidal or suicidal ideation indexed via the structured clinical interview; (h) current psychotropic medication also used in treatment of smoking cessation - due to the possible confounding effects of medication on cessation and related study measures.

Participants smoked $M(S D)=15.2$ (5.1) cigarettes/day. Note that, 3 of the 45 participants needed to re-schedule the experimental session after they reported or biochemical screening indicated that they 
had smoked in the 18-hours prior to the experimental session rather than fully abstaining from smoking as required; each of these participants succeeded to not smoke in their second attempt and then completed session 2. Also, 10 of the 45 participants asked to re-schedule session 2 - this may be due to difficulty in initiating or maintaining the 18-hour smoking deprivation or for unrelated reasons; all of these participants then successfully completed session 2 . Furthermore, 5 additional participants failed to stop smoking for 18-hours (biochemically verified) immediately prior to the experimental session despite 2 attempts - and were thus excluded from participation. Participants received a modest financial honorarium in exchange for their participation.

\section{Procedure}

Session 1: Smoking Status \& Assessment. Participants completed a carbon monoxide (biochemical) analysis to verify smoking status (PPM $\geq 10 ; \mathrm{CMD} / \mathrm{CO}$ Carbon Monoxide Monitor (Model 3110, Spirometrics, Inc. Auburn, ME), a self-report assessment battery and a diagnostic interview not reported here. At the end of session 1, participants were instructed not to smoke during the 18-hours preceding Session 2 and told that their smoking status would be biochemically verified, again, by means of the carbon monoxide monitor upon arrival to session 2 . Sessions 1 and 2 were separated by a minimum of $18 \mathrm{hrs}$ and a maximum of 7 days.

Session 2: Experimental Task Under Deprivation. In session 2, participants first underwent a carbon monoxide analysis to verify smoking abstinence. $\mathrm{CO} \leq 6 \mathrm{PPM}$ indicate that the participant had not smoked in the past 18-hours and eligible for session 2 (Shoptaw et al., 2002). If CO $\geq 6$ ppm, and/or if the participants reported smoking in 18-hours prior to Session 2, then session 2 was rescheduled following another 18-hr deprivation attempt.

Attentional Bias Measurement. The visual emotional dot probe task (MacLeod et al., 1986) was used to measure AB. See Experiment 1 Method for detailed description of the task. AB measurement 
was identical to Experiment 1 but with only one block (500ms stimulus duration), 160 trials, and no surprise trials. Specifically, 15 practice trials were followed by 2 buffer trials (Neutral stimuli), followed by 160 experimental trials, of which 80 trials were NTs, 40 CTs and 40 ITs, randomly distributed across the task. The pictorial stimuli consisted of 20 color photographs of smoking related-scenes (e.g., a man smoking a cigarette, a cigarette beside an ashtray). Each picture was paired with a photograph of another scene matched as closely as possible for content, complexity, color, and luminescence but lacked any smoking-cues. An additional set of 20 picture pairs, with content unrelated to smoking, were used as NTs.

\section{Apparatus}

Stimuli. Pictures were digitized and adjusted in size so that each was 50 x $80 \mathrm{~mm}$ when displayed on the screen. Participants were seated with their eyes about $60 \mathrm{~cm}$ from the screen. When presented side by side on the screen, the distance between the inner edges of the pictures in each pair was $2.75 \mathrm{~cm}$.

Experimental set-up. The experiment was run via E-Prime experimental presentation software (Schneider, Eschman, \& Zuccolotto, 2002). The experimental session was conducted on a HewlettPackard computer and 19" CRT monitor, in an acoustically-insulated room, with a one-way observation window and camera. Participants' responses were measured via Psychology Software Tools Serial Response Box ${ }^{\mathrm{TM}}$.

\section{Results}

Data preparation. $M(S D) \%$ of outlier trials $=1.88(1.05) \%$ and $M(S D) \%$ error trials $=1.05(1.58) \%$ were discarded.

Traditional Computation of Attentional Bias. Sample traditional mean BS (mean IT - mean CT RTs) $M(S D)=13.5(18.8) \mathrm{ms}$. 
Trial-Level Bias Scores (TL-BS): Computation. See Study 1 Results for computation of TL-BS. From a total of $40 \mathrm{IT}$ and $40 \mathrm{CT}, M(S D)$ of computed BSs/participant $=70.1(3.9)$. The $M(S \mathrm{D})$ number of sequential trials with no calculation of TL-BS $=2.1(.10)$. The number of trials between paired IT-CT trials was $M(S D)=2.0(1.15)$. As in Study 1, we visually inspected TL-BS within and across participants (see Figure S1 in the Supplemental Material available online). As predicted, smokingrelated $\mathrm{AB}$ among deprived smokers appears to be expressed in phasic bursts or peaks over time. Furthermore, the magnitude and direction of peaks vary within- and between-participants, as does the degree of temporal variability.

Trial-Level Attentional Bias Quantitative Parameters As in Experiment 1, we computed each of

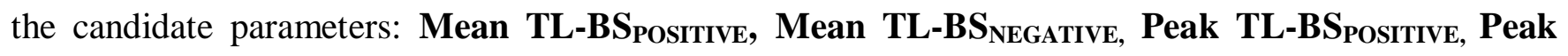

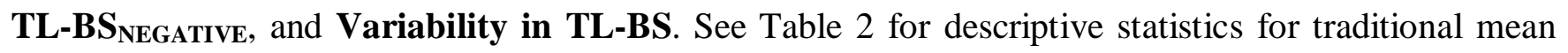
BS and TL-BS parameters.

Split-Half Reliability of Traditional BS and Trial-Level AB Parameters. Split-half reliability is predominantly examined in the field of $\mathrm{AB}$ by randomly splitting task items/trials into two halves (Waechter et al., 2014; Schmukle et al., 2005). However, in the case of the TL-BS approach, as in many other behavioral paradigms (e.g. Implicit Association Task), order of trials in time is crucial. Thus, as in much past work (e.g., Greenwald, Nosek,\& Banaji, 2003), we tested the association between each TLBS parameter from the first-half relative to the second-half of the task. Traditional BS demonstrated very low levels of split-half reliability $\left(r_{\text {Spearman }}=.06\right.$, n.s. $)$, whereas TL-BS parameters demonstrated

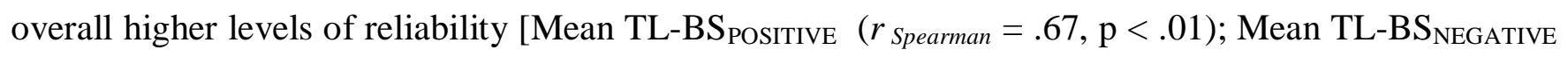
$\left(r_{\text {Spearman }}=.58, \mathrm{p}<.01\right) ;$ Peak TL-BS POSITIVE $_{(}\left(r_{\text {Spearman }}=.44, \mathrm{p}<.01 ;\right.$ Peak TL-BS $\mathrm{NEGATIVE}_{\text {Spearman }}=$ $.31, \mathrm{p}<.05) ;$ TL-BS Variability $\left.\left(r_{\text {Spearman }}=.46, \mathrm{p}<.01\right)\right]$ 
Relations Between Traditional BS and Trial-Level AB Parameters. See Table 2 for zero-order correlations between traditional BS and TL-BS parameters. First, traditional BS demonstrated no significant association with TL-BS away parameters; a small association with TL-BS Variability $(r=$ $.31)$; and only moderate relations with TL-BS towards parameters ( $r$ 's $=.49$ to .55$)$. Second, TL-BS parameters demonstrate moderate to strong relations with one another ( $r^{\prime}$ s $=-.47$ to .82 ). As expected, TL-BS parameters that demonstrate the greatest association are the towards parameters - Mean TL-

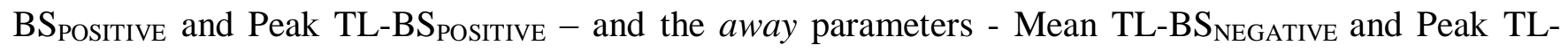
$\mathrm{BS}_{\mathrm{NEGATIVE}}$, respectively.

Cross-Validation of Trial-Level AB Parameters. First, see Table 2 for zero-order correlations between $\mathrm{AB}$ parameters and smoking-related external criterion variable(s) theoretically linked to $\mathrm{AB}-$ daily smoking rate. In summary, higher levels of traditional BS were significantly correlated with smoking rate. Furthermore, as predicted, higher levels of TL-BS towards parameters, Mean TLBS $_{\text {Positive }}$ and Peak TL-BS Positive, were positively correlated with number of cigarettes smoked/day. Second, in a single hierarchical multiple regression analysis, we tested the degree to which TL-BS towards parameters accounted for variance explained by traditional BS as well as incremental, unique significant variance in smoking rate above and beyond traditional BS, and vice-versa. TL-BS towards parameters (Mean TL-BS Positive, Peak TL-BS Positive) explained unique variance in smoking rate beyond the variance explained by traditional BS $\left(F_{(3,41)}=2.5, \Delta r^{2}=.07, p=.04\right)$; traditional BS did not similarly account for unique variance beyond the TL-BS parameters $\left(s r^{2}=.01, p=.25\right)$.

Finally - as in Study 1 - to rule-out the possibility that the observed are a by-product of intraindividual differences and variability in RT - we ran three additional tests. First, as expected, we found that the mean RT on neutral trials was not correlated to smoking rate $(r=.13, \mathrm{n} . \mathrm{s})$. Second, as expected, we found that covarying for mean RT on neutral trials did not effect the reported correlations between 
TL-BS and traditional BS with smoking rate. Third, as in study 1, an additional test of the robustness of TL-BS and derived parameters was done by computing "fake TL-BS" parameters using neutral trials' RTs (see study 1). As expected, parameters derived from this "fake TL-BS" were not correlated with smoking rates $(r=-.12$ to $.18, \mathrm{p}=.24$ to .72$)$.

\section{Experiment 2 Discussion}

We further investigated the expression of $\mathrm{AB}$ with respect to motivationally-relevant appetitive smoking stimuli by means of the proposed TL-BS computational methodology among deprived, daily smokers. First, as in Experiment 1, we found that AB may be operationalized by means of the TL-BS in a manner that provides repeated $\mathrm{AB}$ estimates at the trial-level, temporally concurrent with the dynamic expressions of $\mathrm{AB}$. Second, consistent with prediction, despite the fact that we measured attentional allocation to appetitive and acutely motivationally-relevant smoking cues, TL-BS data were not consistent with a stable, static trait-like signal over time. As in Experiment 1, TL-BS demonstrated phasic bursts of differential attentional allocation over time as well as evidence of fluctuating temporal variability ranging from $\mathrm{AB}$ towards and/or away from smoking images. Moreover, as in Experiment 1, the amplitude and direction of peaks vary within- and between-participants, as does the degree of temporal variability. Third, as in Experiment 1, we were able to derive the same initial candidate parameters reflecting the time-series trial-level $\mathrm{AB}$ expression over time.

We next evaluated the split-half reliability of traditional BS and TL-BS parameters. Traditional BS demonstrated very low levels of reliability relative to significant, albeit modest, reliability of TL-BS parameters. We then evaluated the pattern of relations between traditional mean BSs and TL-BS parameters. We found that traditional BS demonstrated: (a) no association with TL-BS away parameters; (b) a small association with TL-BS Variability; and (c) moderate relations with TL-BS towards parameters. Furthermore, TL-BS parameters demonstrated moderate to strong relations with one 
another. Broadly, these data suggest that traditional mean BS shares a limited degree of variance with TL-BS parameters.

Furthermore, the derived TL-BS parameters permitted us to begin to explore the validity of the TL-BS operationalization of substance-related $\mathrm{AB}$ with respect to a theoretically-relevant external criterion of additive behavior - daily smoking rate. First, as hypothesized, levels of TL-BS POSITIVE $_{\text {. }}$ parameters - reflecting $\mathrm{AB}$ towards smoking stimuli - significantly (concurrently) predicted number of cigarettes smoked daily. Finally, as in Experiment 1, the TL-BS towards parameters explained unique variance in smoking rate above and beyond the effect of traditional mean BS, whereas traditional BS did not explain unique variance in smoking rate beyond the TL-BS towards parameter. Finally, neither mean RT on neutral trials nor "fake TL-BS" computed from neutral were related to smoking rate. Thus, reported TL-BS and parameters are not a by-product or proxy of general variability in RT.

\section{General Discussion}

Attentional biases to motivationally-relevant stimuli are at the heart of a variety of big questions in contemporary clinical psychological science. What are the inter-related roles of emotion, motivation, and information processing in psychopathology? What are the underlying mechanisms, genetic and neural bases of emotional attention and biases of attention? What are the implications of the experimental/clinical modification of these maladaptive biases in attention across levels of analysis (i.e., behavioral, cognitive, neural, epi-genetic)? Accordingly, conceptualizing and operationalizing $\mathrm{AB}$ in a manner that most closely reflects the expression(s) of the phenomena is important theoretically and clinically. In the reported experiments we tested whether the current conceptualization and quantification of $\mathrm{AB}$ reflects the time-series expression and measurement of $\mathrm{AB}$. Moreover, we examined whether the proposed time-series trial-level approach to $\mathrm{AB}$ estimation (TL-BS) provides a 
more valid empirical representation of $\mathrm{AB}$ as well as a means to derive parameters reflecting its dynamics and thereby means to study the phenomenon more precisely.

A number of key findings were observed. First, TL-BS data were not consistent with what we suggest is a core, albeit implicit, assumption of contemporary theory and study of $\mathrm{AB}-$ that $\mathrm{AB}$ expression is a stable, static trait-like signal over time. TL-BS demonstrated phasic bursts of differential attentional allocation over time as well as evidence of fluctuating temporal variability ranging from $\mathrm{AB}$ towards to away from motivationally-relevant aversive and appetitive stimuli. Furthermore, observed individual differences in $\mathrm{AB}$ expression through TL-BSs revealed that the amplitude and direction of peaks vary within- and between-participants, as does the degree of temporal variability. Moreover, we observed that the same participants can express $\mathrm{AB}$ towards and away from target stimuli (Cisler et al., 2009; Zvielli, Bernstein, \& Koster, 2014). One implication of these TL-BS findings is that traditional mean BS may not optimally represent the dynamic expression of $\mathrm{AB}$ in time. Related to these data, traditional BS demonstrated very low levels of split-half reliability in Study 2 (see also Schmukle et al., 2005; Waechter et al., 2014); whereas TL-BS parameters demonstrated - for the first time in this literature - significant albeit modest levels of reliability. These reliability data may be interpreted as further evidence that TL-BS and derived parameters represent a closer representation of the modeled phenomenon of $\mathrm{AB}$ than do traditional mean $\mathrm{BS}$ used to-date.

Of crucial importance, the TL-BS parameters that reflect the time-series trial-level dynamics

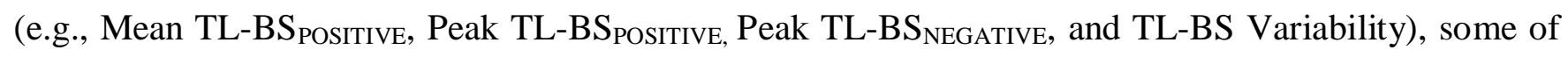
which were unrelated to traditional BS, predicted theoretically-relevant criterion variables (i.e., phobic diagnostic status, smoking rate); and, in most analyses, did so incrementally above and beyond traditional BS estimation. These findings indicate that traditional BS estimation does not likely represent a more valid, "less noisy", estimation of $\mathrm{AB}$ expression in time; nor is it likely that observed TL-BS 
dynamics simply reflect "noise" that straddles a stable and static AB signal as reflected in traditional BS estimation. Were these "dynamics" in time simply noise, then only individual differences in the "true" stable and static signal - reflected by traditional BS - would be expected to demonstrate significant relations with criterion variables.

A number of basic and clinical research implications of the reported data are noteworthy. First, the present findings may have implications for re-evaluating reported $A B$ data as well as designing future studies. Specifically, the various correlates of AB across levels of analysis (self-report, behavioral, neural, genetic) may be mis-estimated (e.g., under-estimated) by extant reliance on traditional BS. One possible future direction may involve re-examination of effects that were null or small in magnitude but that may in fact prove otherwise when AB is modeled in time by TL-BS. It is important to note that the TL-BS computation may be readily preformed on any dot probe task data. Moreover, TL-BS may also be readily preformed for all related tasks (e.g., modified -spatial cueing, visual search, -stroop, etc.) in which $\mathrm{AB}$ is sampled repeatedly over time, in temporally contiguous contrasting trials (e.g., congruent/valid vs. incongruent/invalid vs. neutral). Despite this broad applicability, one key methodological guideline may be taken into consideration in the re-analysis of existing data as well as design of new $A B$ studies wherein TL-BS will be used to quantify $A B$. Specifically, to permit sufficient measurement samples of the temporal dynamics $A B$ and to optimally compute parameters of $\mathrm{AB}$ dynamics, the TL-BS computation necessitates a minimum number of trials (e.g., we estimate at least 40 not including neutral trials); however, to compute split-half reliability of the TL-BS parameters, twice as many trials are likely needed (e.g., 80 trials). Determining the specific number of trials should be the focus of future simulation studies of TL-BS; in the mean time, it may be useful to consider that very short tasks are not optimal for quantifying the temporal dynamics of $A B$ via TL-BS. 
Second, the reported TL-BS dynamics may have novel implications for refining extant theory of AB (e.g., Mogg \& Bradley, 1998; Williams et al., 1988). For example, TL-BS dynamics may help generate novel hypotheses about the mechanisms that may engender and maintain $\mathrm{AB}$ (e.g., Eysenck et al., 2007). For example, future study should test possible processes influencing the dynamics of emotional attention, such as self-monitoring/awareness of attention or attentional (dys)control. For example, awareness of over-engagement with a motivationally-relevant cue may elicit strategic/automatic effort(s) to "down-regulate" emotional responding (Bernstein \& Zvielli, 2014). Moreover, attentional dyscontrol may potentiate a pattern of attentional allocation characterized by dysregulated, dynamic shifts between hypervigilance, over-engagement with motivationally relevant cues, and avoidance of these cues (Cisler et al., 2009).

Third, clinically, conceptualizing and quantifying the dynamics of $A B$ expression by means of TL-BS may prove important to a variety of core questions at the heart information-processing in psychopathology and related efforts to therapeutically target $\mathrm{AB}$. For example, modeling $\mathrm{AB}$ by means of TL-BS may permit a more refined and valid means to explore the components and underlying mechanisms of $\mathrm{AB}$ that may indeed reflect vulnerability for or proxies of psychopathology (e.g., threatrelated $\mathrm{AB}$ in anxiety psychopathology). Relatedly, modeling the dynamics of $\mathrm{AB}$ in time may permit a more direct and potentially rigorous means to evaluate of the nature of change(s) that various nonspecific interventions (e.g., cognitive-behavior therapy, pharmacotherapy) and emerging specific attentional bias modification interventions - Attention Bias Modification (ABM; Heeren et al., 2013), Attention Feedback Awareness and Control Training (A-FACT; Bernstein \& Zvielli, 2014), or attentional control training (e.g., Wiers, Gladwin, Hofmann, Salemink, \& Ridderinkhof, 2013) - may have on AB. Indeed, because studies to-date relied on traditional BS that may misrepresent the dynamics of $\mathrm{AB}$ expression, re-analysis of published and unpublished data may help disambiguate mixed findings 
and the ongoing debate regarding the efficacy and size of effects of ABM (Beard et al., 2012; Hallion \& Ruscio, 2011). Furthermore, clinically, AB dynamics seen in a time-series trial-level approach to operationalizing $\mathrm{AB}$ expression may furthermore help shape how training could be more optimally delivered. An example of this type of effort is reflected in our recent development of Attention Feedback Awareness and Control Training (A-FACT; Bernstein \& Zvielli, 2014), grounded in the theoretical and empirical model driving the present TL-BS approach. Accordingly, A-FACT delivers feedback regarding a person's (biased) allocation of attention concurrent with its expression in real-time. In light of the present TL-BS findings, such intervention paradigms sensitive to the dynamics of $A B$ may be a promising new direction for attentional training.

There are a number of methodological considerations and limitations of the reported experiments. First, we explored TL-BS in the modified dot probe only. Future work evaluating the degree to which the observed findings generalize across various paradigms (e.g., modified spatial cueing) is important. Similarly, our tests were limited to "emotional" attention. Other studies could test TL-BS and evaluate similar dynamics in non-emotional attentional processes (Pashler, 1998). This type of work may be important to understand the role of emotion, motivation, and context in the observed dynamics of TL-BS estimation of AB expression. Second, validation analyses were cross-sectional limiting our capacity to infer directionality or causality between TL-BS parameters of AB and diagnostic status or smoking rate. To build on these initial findings, prospective data may be important to examine questions of directionality and experimental work to examine questions of causality. Third, we began to test whether observed temporal dynamics of $\mathrm{AB}$ - observed via TL-BS - may help account for questionable psychometrics of modified dot probe data and specifically traditional mean bias scores (e.g., Schmukle, 2005). Though we were able to examine the split-half reliability of traditional mean bias scores and the novel TL-BS parameters in Experiment 2, we were not able to do so for Experiment 
1 due to an insufficient number of trials. We believe that future large-scale psychometric study of TLBS and parameters is important. Fourth, as highlighted in the Introduction, the computational methodology used to estimate TL-BS or AB in time in the reported experiments represent only one of multiple potential related approaches. Real- and simulated- data may be useful in further evaluating the present TL-BS approach relative to alternative computational methods and parameters to measure and illuminate $\mathrm{AB}$ in time.,

In summary, we hope that the proposed approach to the conceptualization and study of $\mathrm{AB}$ in time may help bring the conceptualization and quantification of $\mathrm{AB}$ closer to the nature of the phenomenon - and that this may ultimately help advance basic and clinical research. In so far as the proposed time-series trial-level approach to the computation of $\mathrm{AB}$ may better reflect the phenomenon(a), then we hope that it may ultimately help advance understanding of this bio-psychobehavioral process(es) and its role in human (mal)adaptation. 
Table 1

Trial-Level Bias Score (TL-BS) Parameters Above and Beyond Traditional Bias Score Predicting Diagnostic Status

\begin{tabular}{|c|c|c|c|c|c|c|c|c|c|c|c|c|}
\hline \multicolumn{7}{|c|}{$500 \mathrm{~ms}$} & \multicolumn{6}{|c|}{$200 \mathrm{~ms}$} \\
\hline & $\begin{array}{c}\text { Spider Phobia } \\
\text { (N=41) }\end{array}$ & $\begin{array}{l}\text { Control } \\
(\mathbf{N}=\mathbf{2 5})\end{array}$ & $O R^{\dagger}$ & $(95 \% C I)$ & $p$ & $r^{2}$ & $\begin{array}{r}\text { Spider Phobia } \\
\qquad M(\end{array}$ & Control & $\boldsymbol{O R}^{\dagger}$ & $(95 \% C I)$ & $p$ & $r^{2}$ \\
\hline$\underline{\text { Step 1* }}$ & & & & & & & & & & & & \\
\hline Traditional Mean BS & $22.9(57.7)$ & $3.5(28.7)$ & 1.11 & $(0.99-1.24)$ & .07 & .06 & $28.3(67.1)$ & $1.0(29.5)$ & 1.15 & $(1.02-1.30)$ & .03 & .10 \\
\hline$\frac{\frac{\text { Step } 1}{\text { Mean TL-BS }}}{\text { PositivE }}$ & $93.0(64.5)$ & $61.2(27.5)$ & 1.19 & $(1.04-1.37)$ & .02 & 13 & $105.0(68.5)$ & $65.3(28.4)$ & 1.26 & $(1.08-1.47)$ & .01 & .20 \\
\hline$\frac{\text { Step } 2}{\text { Traditional Mean BS }}$ & & & 096 & $(081-1,14)$ & 35 & & & & 112 & $(0$ 97-1 29) & 10 & \\
\hline Mean TL-BS Positive & & & 1.23 & $(1.02-1.48)$ & .04 & .08 & & & 1.22 & $(1.05-1.42)$ & .01 & .13 \\
\hline$\frac{\text { Step } 1}{\text { Mean TL-BS }}$ & $-83.2(53.0)$ & $-59.6(38.2)$ & 0.88 & $(0.77-0.99)$ & .04 & .09 & $-85.7(58.3)$ & $-60.5(38.4)$ & 0.88 & $(0.79-0.99)$ & .04 & .08 \\
\hline$\frac{\text { Step 1 }}{\text { Peak TL-BS Positive }}$ & $276.6(171.0)$ & $194.6(140.3)$ & 1.04 & $(1.005-1.07)$ & .03 & .09 & $311.2(224.1)$ & $198.9(125.9)$ & 1.04 & $(1.01-1.07)$ & .02 & .12 \\
\hline$\frac{\text { Step 2 }}{\text { Traditional Mean BS }}$ & & & 1.05 & $(0.92-1.19)$ & .28 & & & & 1.14 & $(0.996-1.30)$ & .06 & \\
\hline Peak TL-BS Positive & & & 1.03 & $(0.99-1.07)$ & .10 & .04 & & & 1.03 & $(1.003-1.06)$ & .04 & .07 \\
\hline$\frac{\text { Step } 1}{\text { Peak TL-BS }}$ & $-214.1(163.6)$ & $-179.4(170.0)$ & 0.99 & $(0.96-1.01)$ & .21 & .02 & $-213.7(155.2)$ & $-216.1(186.6)$ & 1.00 & $(0.98-1.03)$ & .48 & .00 \\
\hline$\frac{\text { Step } 1}{\text { TL-BS Variability }}$ & $34.9(15.8)$ & $25.0(9.6)$ & 1.90 & $(1.25-2.88)$ & .01 & .17 & $38.6(26.2)$ & $25.9(7.1)$ & 1.65 & $(1.12-2.42)$ & .02 & .15 \\
\hline$\frac{\text { Step } 2}{\text { Traditional Mean BS }}$ & & & 1.08 & $(0.95-1.24)$ & .16 & & & & 1.20 & $(1.03-1.40)$ & .02 & \\
\hline TL-BS Variability & & & 1.83 & $(1.19-2.81)$ & .01 & .13 & & & 1.55 & $(1.08-2.22)$ & .02 & .13 \\
\hline
\end{tabular}

Note. TL- Trial Level. BS- Bias Score. ${ }^{\dagger}$ Odds Ratio was computed such that likelihood in phobic versus control group status reflects 10 units of change (10ms) in traditional and TL-BS independent variables. $r^{2}=$ Nagelkerke's $r^{2}$. Significance presented is for one-tailed tests. Nagelkerke's incremental $r^{2}$ for step 2 reflects variance in diagnostic status risk explained by the TL-BS parameter above and beyond risk explained by Traditional Mean BS at step 1. * Only a single step analysis is reported for Traditional Mean BS because the incremental contributions of the TL-BS parameters beyond Traditional Mean BS are reported in step 2 in each of the subsequent 2-step analyses reported in the table. 
Table 2

Zero-Order Correlations Between Traditional Bias Scores, Trial-Level Bias Score Parameters and Daily Smoking Rate

\begin{tabular}{|c|c|c|c|c|c|c|c|c|c|}
\hline & 1 & 2 & 3 & 4 & 5 & 6 & 7 & $\mathbf{M}(\mathbf{S D})$ & Range \\
\hline 1. Traditional Mean BS & 1 & $.55^{* *}$ & -.16 & $.49^{* *}$ & .02 & $.31 *$ & $.29 *$ & $13.5(18.8)$ & $-12.5-67.1$ \\
\hline 2. Mean TL-BS & & 1 & $-.56^{* *}$ & $.71 * *$ & $-.55^{* *}$ & $.71 * *$ & $.32 *$ & $67.9(24.4)$ & $23.6-126.0$ \\
\hline 3. Mean TL-BS NEGATIVE & & & 1 & $-.58 * *$ & $.82 * *$ & $-.59^{*}$ & -.12 & $-61.5(23.3)$ & $-107.2--23.0$ \\
\hline 4. Peak TL-BS Positive & & & & 1 & $-.47 * *$ & $.64 * *$ & $.37 * *$ & $212.8(101.2)$ & $51.0-556.0$ \\
\hline 5. Peak TL-BS ${ }_{\text {NEGative }}$ & & & & & 1 & $-.54 * *$ & .01 & $-176.8(76.5)$ & $-379.0--62.0$ \\
\hline 6. TL-BS Variability & & & & & & 1 & .10 & $26.3(9.0)$ & $8.7-51.7$ \\
\hline 7. \# Cigarettes/Day & & & & & & & 1 & $15.2(5.1)$ & $10.0-30.0$ \\
\hline
\end{tabular}

Note. $* p<0.05, * * p<0.01$. 


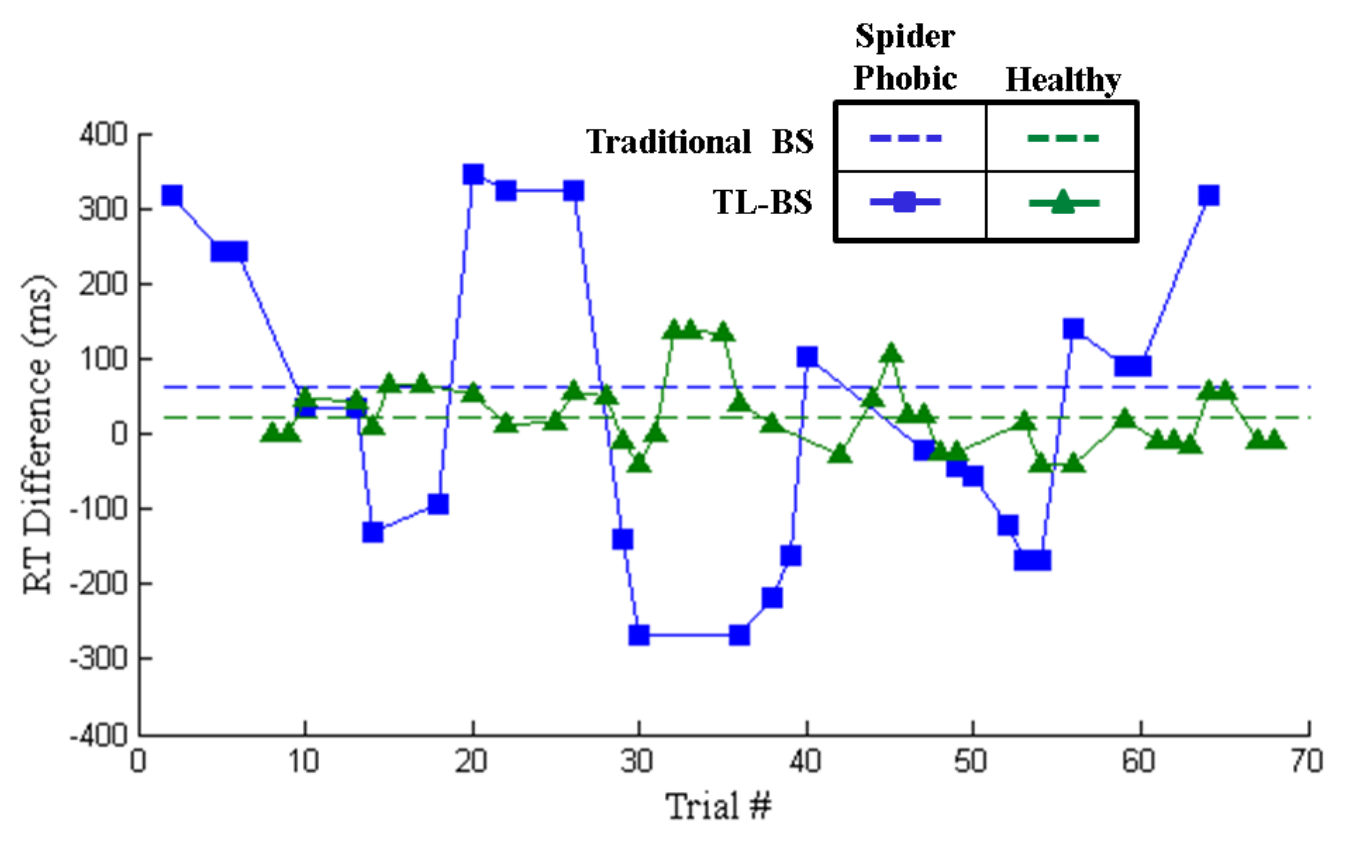

Figure 1. Traditional Mean and Trial-Level Bias Scores for One Spider Phobic Participant and One Healthy Control Participant.

Note . RT = Response Time; Traditional BS = Traditional Bias Score (Mean RT of ITs- CTs); TL-BS = Trial Level Bias Scores (Adjacent IT - CT pairs). 


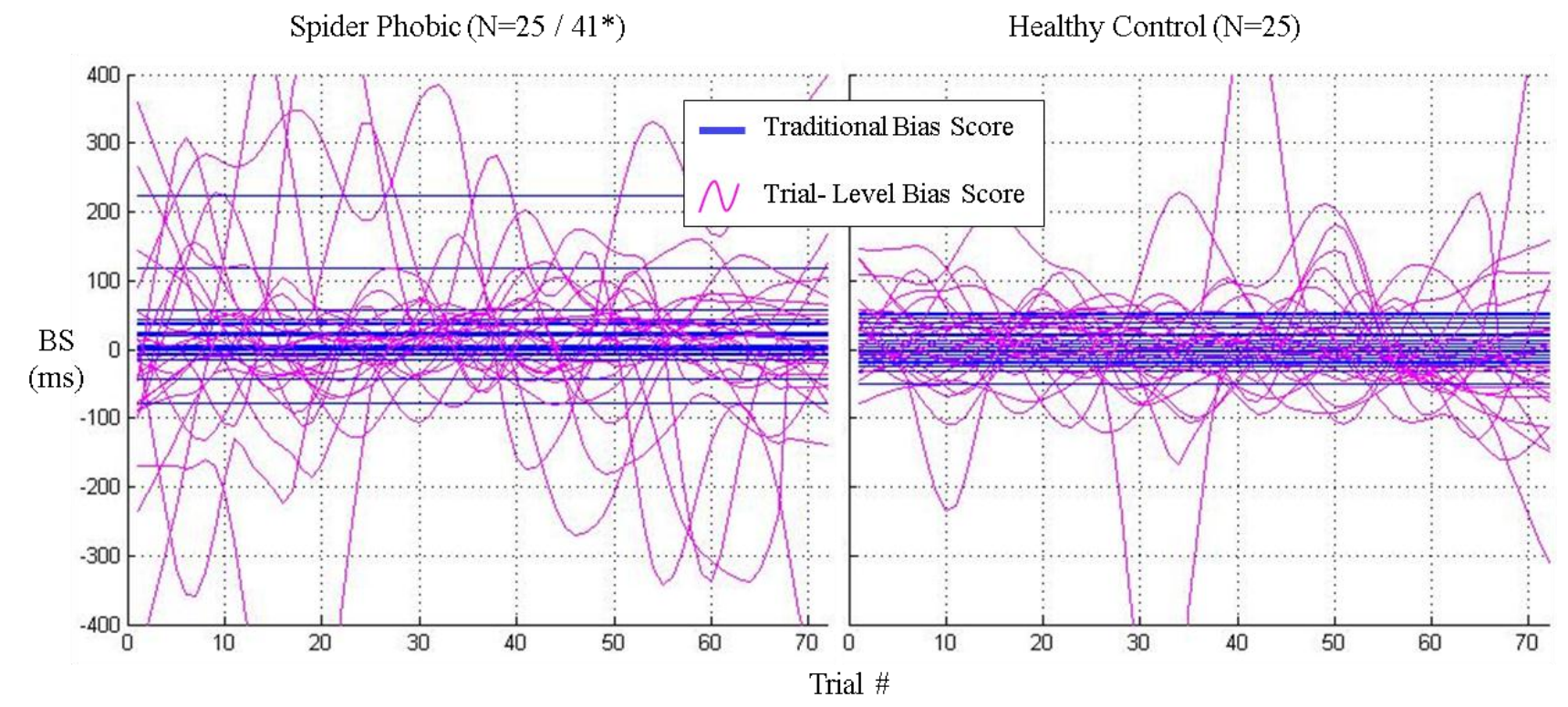

Figure 2. Traditional and Trial-Level Bias Scores: Spaghetti Plots by Diagnostic Status.

Note. ${ }^{*} \mathrm{~N}=25$ of the 41 spider phobic participants were randomly selected to facilitate comparison with $\mathrm{N}=25$ control group. TL-BS were interpolated to 70 data-points and smoothed by a running mean with a 10-trials window size (Stimulus Duration $=500 \mathrm{~ms}$ ). This was done only for the purpose of visualizing spaghetti plots. All reported analyses in the text were performed on the $\mathrm{N}=41$ spider phobics and $\mathrm{N}=25$ control participants. 
Acknowledgements: Dr. Bernstein recognizes the funding support from the Israeli Council for Higher Education Yigal Alon Fellowship, the European Union FP-7 Marie Curie Fellowship International Reintegration Grant, Psychology Beyond Borders Mission Award, Israel Science Foundation, the University of Haifa Research Authority Exploratory Grant, and the Rothschild-Caesarea Foundation's Returning Scientists Project at the University of Haifa. Mr. Zvielli recognizes the support from the University of Haifa President's Doctoral Fellowship Program. 


\section{References}

American Psychiatric Association. (2013). Diagnostic and statistical manual of mental disorders: DSM 5 Books4US.

Ardid, S., Wang, X. J., Gomez-Cabrero, D., \& Compte, A. (2010). Reconciling coherent oscillation with modulation of irregular spiking activity in selective attention: Gamma-range synchronization between sensory and executive cortical areas. The Journal of Neuroscience, 30(8), 2856-2870. doi:10.1523/JNEUROSCI.4222-09.2010; 10.1523/JNEUROSCI.4222-09.2010

Armstrong, T., \& Olatunji, B. O. (2012). Eye tracking of attention in the affective disorders: A metaanalytic review and synthesis. Clinical Psychology Review, 32(8), 704-723.

Baker, T. B., Piper, M. E., McCarthy, D. E., Majeskie, M. R., \& Fiore, M. C. (2004b). Addiction motivation reformulated: An affective processing model of negative reinforcement. Psychological Review, 111(1), 33-51. doi:10.1037/0033-295X.111.1.33

Bar-Haim, Y., Holoshitz, Y., Eldar, S., Frenkel, T. I., Muller, D., Charney, D. S., . . Wald, I. (2010). Life-threatening danger and suppression of attention bias to threat. American Journal of Psychiatry, 167(6), 694-698.

Bar-Haim, Y., Lamy, D., Pergamin, L., Bakermans-Kranenburg, M., \& van IJzendoorn, M. H. (2007). Threat-related attentional bias in anxious and nonanxious individuals: A meta-analytic study. Psychological Bulletin, 133(1), 1-24. doi:10.1037/0033-2909.133.1.1

Beard, C., Sawyer, A. T., \& Hofmann, S. G. (2012). Efficacy of attention bias modification using threat and appetitive stimuli: A meta-analytic review. Behavior Therapy, 43(4), 724-740. 
Beevers, C. G., Wells, T. T., Ellis, A. J., \& McGeary, J. E. (2009). Association of the serotonin transporter gene promoter region (5-HTTLPR) polymorphism with biased attention for emotional stimuli. Journal of Abnormal Psychology, 118(3), 670.

Bernstein, A., \& Zvielli, A. (2014). Attention Feedback Awareness and Control Training (A-FACT): Experimental test of a novel intervention paradigm targeting attentional bias. Behaviour research and therapy, 55, 18-26.

Bishop, S. J. (2008). Trait anxiety and impoverished prefrontal control of attention. Nature Neuroscience, 12(1), 92-98.

Bishop, S., Duncan, J., Brett, M., \& Lawrence, A. D. (2004). Prefrontal cortical function and anxiety: Controlling attention to threat-related stimuli. Nature Neuroscience, 7(2), 184-188.

Bouman, T., de Ruiter, C., \& Hoogduin, C. (1995). Dutch Version of the Anxiety Disorders Interview Schedule for DSM-IV,

Bradley, B. P., Mogg, K., \& Millar, N. H. (2000). Covert and overt orienting of attention to emotional faces in anxiety. Cognition \& Emotion, 14(6), 789-808.

Brosschot, J. F., de Ruiter, C., \& Kindt, M. (1999). Processing bias in anxious subjects andrepressors, measured by emotional stroop interferenceandattentional allocation. Personality and Individual Differences, 26(5), 777-793.

Brown, T. A., DiNardo, P., \& Barlow, D. H. (1994). Anxiety disorders interview schedule for DSM-IV.: Client interview schedule Oxford University Press. 
Browning, M., Holmes, E. A., Murphy, S. E., Goodwin, G. M., \& Harmer, C. J. (2010). Lateral prefrontal cortex mediates the cognitive modification of attentional bias. Biological Psychiatry, $67(10), 919-925$.

Cisler, J. M., Bacon, A. K., \& Williams, N. L. (2009). Phenomenological characteristics of attentional biases towards threat: A critical review. Cognitive Therapy and Research, 33(2), 221-234.

Cisler, J. M., \& Koster, E. H. (2010). Mechanisms of attentional biases towards threat in the anxiety disorders: An integrative review. Clinical Psychology Review, 30(2), 203.

Clark, D. M., \& Wells, A. (1995). A cognitive model of social phobia. Social Phobia: Diagnosis, Assessment, and Treatment, 41(68), 00022-00023.

Clarke, P. J., Chen, N., \& Guastella, A. J. (2012). Prepared for the best: Readiness to modify attentional processing and reduction in anxiety vulnerability in response to therapy. Emotion, 12(3), 487.

Clarke, P. J., MacLeod, C., \& Guastella, A. J. (2013). Assessing the role of spatial engagement and disengagement of attention in anxiety-linked attentional bias: A critique of current paradigms and suggestions for future research directions. Anxiety, Stress \& Coping, 26(1), 1-19.

Cooper, R. M., \& Langton, S. R. (2006). Attentional bias to angry faces using the dot-probe task? it depends when you look for it. Behaviour Research and Therapy, 44(9), 1321-1329.

Dalgleish, T., Taghavi, R., Neshat-Doost, H., Moradi, A., Canterbury, R., \& Yule, W. (2003). Patterns of processing bias for emotional information across clinical disorders: A comparison of attention, memory, and prospective cognition in children and adolescents with depression, generalized anxiety, 
and posttraumatic stress disorder. Journal of Clinical Child and Adolescent Psychology, 32(1), 1021.

De Raedt, R., \& Koster, E. H. (2010). Understanding vulnerability for depression from a cognitive neuroscience perspective: A reappraisal of attentional factors and a new conceptual framework. Cognitive, Affective, \& Behavioral Neuroscience, 10(1), 50-70.

Dear, B. F., Sharpe, L., Nicholas, M. K., \& Refshauge, K. (2011). Pain-related attentional biases: The importance of the personal relevance and ecological validity of stimuli. The Journal of Pain, 12(6), $625-632$.

Derryberry, D., \& Reed, M. A. (2002). Anxiety-related attentional biases and their regulation by attentional control. Journal of Abnormal Psychology, 111(2), 225.

Desimone, R., \& Duncan, J. (1995). Neural mechanisms of selective visual attention. Annual Review of Neuroscience, 18(1), 193-222.

Eldar, S., Yankelevitch, R., Lamy, D., \& Bar-Haim, Y. (2010). Enhanced neural reactivity and selective attention to threat in anxiety. Biological Psychology, 85(2), 252-257.

Eysenck, M. W., Derakshan, N., Santos, R., \& Calvo, M. G. (2007). Anxiety and cognitive performance: Attentional control theory. Emotion, 7(2), 336.

Field, M., Munafò, M. R., \& Franken, I. H. (2009). A meta-analytic investigation of the relationship between attentional bias and subjective craving in substance abuse. Psychological Bulletin, 135(4), 589. 
Fox, E., Russo, R., Bowles, R., \& Dutton, K. (2001). Do threatening stimuli draw or hold visual attention in subclinical anxiety? Journal of Experimental Psychology: General, 130(4), 681.

Fox, E., Zougkou, K., Ridgewell, A., \& Garner, K. (2011). The serotonin transporter gene alters sensitivity to attention bias modification: Evidence for a plasticity gene. Biological Psychiatry, 70(11), 1049-1054.

Greenwald, A. G., Nosek, B. A., \& Banaji, M. R. (2003). Understanding and using the implicit association test: I. An improved scoring algorithm. Journal of personality and social psychology, $85(2), 197-216$.

Hallett, P. (1978). Primary and secondary saccades to goals defined by instructions. Vision Research, $18(10), 1279-1296$.

Hallion, L. S., \& Ruscio, A. M. (2011). A meta-analysis of the effect of cognitive bias modification on anxiety and depression. Psychological Bulletin, 137(6), 940-958. doi:10.1037/a0024355

Harris, C. R., \& Pashler, H. (2004). Attention and the processing of emotional words and names: Not so special after all. Psychological Science, 15(3), 171-178.

Heeren, A., De Raedt, R., Koster, E. H., \& Philippot, P. (2013). The (neuro) cognitive mechanisms behind attention bias modification in anxiety: Proposals based on theoretical accounts of attentional bias. Frontiers in Human Neuroscience, 7

Herrmann, C. S., \& Knight, R. T. (2001). Mechanisms of human attention: Event-related potentials and oscillations. Neuroscience \& Biobehavioral Reviews, 25(6), 465-476. 
Insel, T., Cuthbert, B., Garvey, M., Heinssen, R., Kozak, M., Pine, D., \& Wang, P. (2010). Research domain criteria (RDoC): Developing a valid diagnostic framework for research on mental disorders. American Journal of Psychiatry, 167, 748-751.

Koster, E. H. W., Crombez, G., Verschuere, B., Van Damme, S., \& Wiersema, J. -. (2006). Components of attentional bias to threat in high trait anxiety: Facilitated engagement, impaired disengagement, and attentional avoidance. Behaviour Research and Therapy, 44, 1757-1771. doi:10.1037/a0014379

Lang, P. J., Bradley, M. M., \& Cuthbert, B. N. (1997). Motivated attention: Affect, activation, and action. Attention and Orienting: Sensory and Motivational Processes, , 97-135.

Lavy, E., Van den Hout, M., \& Arntz, A. (1993). Attentional bias and spider phobia: Conceptual and clinical issues. Behaviour Research and Therapy, 31(1), 17-24.

Luijten, M., Veltman, D. J., Hester, R., Smits, M., Pepplinkhuizen, L., \& Franken, I. H. (2012). Brain activation associated with attentional bias in smokers is modulated by a dopamine antagonist. Neuropsychopharmacology, 37(13), 2772-2779.

MacLeod, C., \& Mathews, A. (1988). Anxiety and the allocation of attention to threat. The Quarterly journal of experimental psychology, 40(4), 653-670.

MacLeod, C., Mathews, A., \& Tata, P. (1986). Attentional bias in emotional disorders. Journal of Abnormal Psychology, 95(1), 15.

Mathews, A., \& MacLeod, C. (2005). Cognitive vulnerability to emotional disorders. Annu.Rev.Clin.Psychol., 1, 167-195. 
McKenna, F. P., \& Sharma, D. (2004). Reversing the emotional Stroop effect: The role of fast and slow components. Journal of Experimental Psychology: Learning, Memory, and Cognition, 30(2), 382392.

Mogg, K., Bradley, B. P., Dixon, C., Fisher, S., Twelftree, H., \& McWilliams, A. (2000). Trait anxiety, defensiveness and selective processing of threat: An investigation using two measures of attentional bias. Personality and Individual Differences, 28(6), 1063-1077.

Mogg, K., Holmes, A., Garner, M., \& Bradley, B. P. (2008). Effects of threat cues on attentional shifting, disengagement and response slowing in anxious individuals. Behaviour Research and Therapy, 46(5), 656-667.

Mogg, K., McNamara, J., Powys, M., Rawlinson, H., Seiffer, A., \& Bradley, B. P. (2000). Selective attention to threat: A test of two cognitive models of anxiety. Cognition \& Emotion, 14(3), 375-399.

Mogg, K., \& Bradley, B. P. (1998). A cognitive-motivational analysis of anxiety. Behaviour Research and Therapy, 36(9), 809-848.

Mogg, K., \& Bradley, B. P. (2006). Time course of attentional bias for fear-relevant pictures in spiderfearful individuals. Behaviour Research and Therapy, 44(9), 1241-1250.

Mogg, K., Bradley, B., Miles, F., \& Dixon, R. (2004). BRIEF REPORT time course of attentional bias for threat scenes: Testing the vigilance-avoidance hypothesis. Cognition and Emotion, 18(5), 689700. 
Monk, C., Nelson, E., McClure, E., Mogg, K., Bradley, B., Leibenluft, E., . . Ernst, M. (2006). Ventrolateral prefrontal cortex activation and attentional bias in response to angry faces in adolescents with generalized anxiety disorder. American Journal of Psychiatry, 163(6), 1091-1097.

Muris, P., \& Merckelbach, H. (1996). A comparison of two spider fear questionnaires. Journal of Behavior Therapy and Experimental Psychiatry, 27(3), 241-244.

Öhman, A., Flykt, A., \& Esteves, F. (2001). Emotion drives attention: Detecting the snake in the grass. Journal of Experimental Psychology: General, 130(3), 466.

Öhman, A., Flykt, A., \& Lundqvist, D. (2000). Unconscious emotion: Evolutionary perspectives, psychophysiological data and neuropsychological mechanisms. Cognitive Neuroscience of Emotion, 296

Öhman, A., \& Mineka, S. (2001). Fears, phobias, and preparedness: Toward an evolved module of fear and fear learning. Psychological Review; Psychological Review, 108(3), 483-522. doi:10.1037/0033295X.108.3.483

Osinsky, R., Lösch, A., Hennig, J., Alexander, N., \& MacLeod, C. (2012). Attentional bias to negative information and 5-HTTLPR genotype interactively predict students' emotional reactivity to first university semester. Emotion, 12(3), 460.

Pashler, H. E. (1998). The psychology of attention MIT press Cambridge, MA.

Perez-Edgar, K., Bar-Haim, Y., McDermott, J. M., Gorodetsky, E., Hodgkinson, C. A., Goldman, D., . . . Fox, N. A. (2010). Variations in the serotonin-transporter gene are associated with attention bias patterns to positive and negative emotion faces. Biological Psychology, 83(3), 269-271. 
Pflugshaupt, T., Mosimann, U. P., Wartburg, R. v., Schmitt, W., Nyffeler, T., \& Müri, R. M. (2005). Hypervigilance-avoidance pattern in spider phobia. Journal of Anxiety Disorders, 19(1), 105-116.

Raes, A. K., Koster, E. H., Loeys, T., \& De Raedt, R. (2011). Pathways to change in one-session exposure with and without cognitive intervention: An exploratory study in spider phobia. Journal of Anxiety Disorders, 25(7), 964-971.

Robinson, O. J., Charney, D. R., Overstreet, C., Vytal, K., \& Grillon, C. (2012). The adaptive threat bias in anxiety: Amygdala-dorsomedial prefrontal cortex coupling and aversive amplification. NeuroImage, 60(1), 523-529.

Schmukle, S. C. (2005). Unreliability of the dot probe task. European Journal of Personality, 19(7), 595-605.

Schneider, W., Eschman, A., \& Zuccolotto, A. (2002). E-prime: User's guide Psychology Software Incorporated.

Shechner, T., Britton, J. C., Pérez-Edgar, K., Bar-Haim, Y., Ernst, M., Fox, N. A., . . Pine, D. S. (2012). Attention biases, anxiety, and development: Toward or away from threats or rewards? Depression and Anxiety, 29(4), 282-294.

Sheppes, G., Luria, R., Fukuda, K., \& Gross, J. J. (2013). There's more to anxiety than meets the eye: Isolating threat-related attentional engagement and disengagement biases. Emotion, 13(3), 520.

Shoptaw, S., Rotheram-Fuller, E., Yang, X., Frosch, D., Nahom, D., Jarvik, M. E., . . Ling, W. (2002). Smoking cessation in methadone maintenance. Addiction, 97(10), 1317-1328. 
Siegle, G. J., Ghinassi, F., \& Thase, M. E. (2007). Neurobehavioral therapies in the 21st century:

Summary of an emerging field and an extended example of cognitive control training for depression. Cognitive Therapy and Research, 31(2), 235-262.

Szymanski, J., \& O'Donohue, W. (1995). Fear of spiders questionnaire. Journal of Behavior Therapy and Experimental Psychiatry, 26(1), 31-34.

Van Bockstaele, B., Verschuere, B., Crombez, G., De Houwer, J., Tibboel, H., \& Koster, E. (2014). A review of current evidence for the causal impact of attentional bias on fear and anxiety. Psychological Bulletin 140, 682-721

Van Bockstaele, B., Koster, E. H., Verschuere, B., Crombez, G., \& De Houwer, J. (2012). Limited transfer of threat bias following attentional retraining. Journal of Behavior Therapy and Experimental Psychiatry, 43(2), 794-800.

Van Bockstaele, B., Verschuere, B., Tibboel, H., De Houwer, J., Crombez, G., \& Koster, E. H. (2013). A review of current evidence for the causal impact of attentional bias on fear and anxiety.

Waechter, S., Nelson, A. L., Wright, C., Hyatt, A., \& Oakman, J. (2014). Measuring attentional bias to threat: Reliability of dot probe and eye movement indices. Cognitive Therapy and Research, 38(3), 313-333.

Waters, A. J., Shiffman, S., Sayette, M. A., Paty, J. A., Gwaltney, C. J., \& Balabanis, M. H. (2003). Attentional bias predicts outcome in smoking cessation. Health Psychology, 22(4), 378. 
Weierich, M., \& Barrett, L. F. (2010). Affect is a source of visual attention. psychologists have made a distinction between "affect" and "cognition," suggesting that the latter operates to. Social Psychology of Visual Perception, , 125.

Wiers, R. W., Gladwin, T. E., Hofmann, W., Salemink, E., \& Ridderinkhof, K. R. (2013). Cognitive bias modification and cognitive control training in addiction and related psychopathology mechanisms, clinical perspectives, and ways forward. Clinical Psychological Science, 1(2), 192-212.

Williams, J. M. G., Watts, F. N., MacLeod, C., \& Mathews, A. (1988). Cognitive psychology and emotional disorders. John Wiley \& Sons.

Williams, J. M. G., Mathews, A., \& MacLeod, C. (1996). The emotional stroop task and psychopathology. Psychological Bulletin, 120(1), 3-24.

Wilson, E., \& MacLeod, C. (2003). Contrasting two accounts of anxiety-linked attentional bias: Selective attention to varying levels of stimulus threat intensity. Journal of Abnormal Psychology, 112(2), 212.

Wolfe, J. M. (1994). Guided search 2.0 a revised model of visual search. Psychonomic Bulletin \& Review, 1(2), 202-238.

Yiend, J., \& Mathews, A. (2001). Anxiety and attention to threatening pictures. The Quarterly Journal of Experimental Psychology: Section A, 54(3), 665-681.

Zvielli A., Bernstein A., \& Koster, E.H.W. (2014). Dynamics of Attentional Bias to Threat in Anxious Adults: Bias towards and/or Away? PLoS ONE 9(8), e104025. doi:10.1371/journal.pone.0104025 
Temporal Dynamics of Attentional Bias 47 


\section{Footnotes}

${ }^{i}$ We chose 5 trials following pilot testing and pragmatic effort to maximize the number of possible ITCT pairs to generate a maximally stable time-series plot of TL-BSs, yet ensure that temporal contiguity between CTs and ITs is maximized. The latter is fundamental to the time-series assumption of $\mathrm{AB}$ expression and the assumption that two temporally contiguous trials (e.g., trial 8 and 9) are more likely reflective of the same AB signal than are two more temporally distal trials (e.g., trial 8 and trial 18). See Analysis 1 in the Supplemental Material available online for further details regarding 5-trial window length.

ii There are, of course, a variety of possible methodological permutations of the proposed TL-BS computation (e.g., CT and/or IT trials relative to temporally contiguous NT trials; IT-CT matching > or $<$ than 5 trials away from target trial, etc.). 